\title{
Uncertainty relations for generalized metric adjusted skew information and generalized metric adjusted correlation measure
}

\author{
Kenjiro Yanagi ${ }^{1 *}$, Shigeru Furuichi ${ }^{2}$ and Ken Kuriyama ${ }^{3}$
}

*Correspondence:
yanagi@yamaguchi-u.ac.jp
${ }^{1}$ Graduate School of Science and
Engineering, Yamaguchi University,
Une, Yamaguchi 755-8611, Japan
Full list of author information is
available at the end of the article

available at the end of the article

\begin{abstract}
In this paper, we give a Heisenberg-type or a Schrödinger-type uncertainty relation for generalized metric adjusted skew information or generalized metric adjusted correlation measure. These results generalize the previous result of Furuichi and Yanagi (J. Math. Anal. Appl. 388:1147-1156, 2012).
\end{abstract}

AMS: Primary: 15A45, 47A63; secondary: 94A17

Keywords: Trace inequality; Metric adjusted skew information;

Metric adjusted correlation measure

\section{Introduction}

We start from the Heisenberg uncertainty relation [1]:

$$
V_{\rho}(A) V_{\rho}(B) \geq \frac{1}{4}|\operatorname{Tr}[\rho[A, B]]|^{2}
$$

for a quantum state (density operator) $\rho$ and two observables (self-adjoint operators) $A$ and $B$. The further stronger result was given by Schrödinger in $[2,3]$ :

$$
V_{\rho}(A) V_{\rho}(B)-\left|\operatorname{Re}\left\{\operatorname{Cov}_{\rho}(A, B)\right\}\right|^{2} \geq \frac{1}{4}|\operatorname{Tr}[\rho[A, B]]|^{2},
$$

where the covariance is defined by $\operatorname{Cov}_{\rho}(A, B) \equiv \operatorname{Tr}[\rho(A-\operatorname{Tr}[\rho A] I)(B-\operatorname{Tr}[\rho B] I)]$.

The Wigner-Yanase skew information represents a measure for non-commutativity between a quantum state $\rho$ and an observable $H$. Luo introduced the quantity $U_{\rho}(H)$ representing a quantum uncertainty excluding the classical mixture [4]:

$$
U_{\rho}(H) \equiv \sqrt{V_{\rho}(H)^{2}-\left(V_{\rho}(H)-I_{\rho}(H)\right)^{2}},
$$

with the Wigner-Yanase skew information [5]:

$$
I_{\rho}(H) \equiv \frac{1}{2} \operatorname{Tr}\left[\left(i\left[\rho^{1 / 2}, H_{0}\right]\right)^{2}\right]=\operatorname{Tr}\left[\rho H_{0}^{2}\right]-\operatorname{Tr}\left[\rho^{1 / 2} H_{0} \rho^{1 / 2} H_{0}\right], \quad H_{0} \equiv H-\operatorname{Tr}[\rho H] I,
$$

and then he successfully showed a new Heisenberg-type uncertainty relation on $U_{\rho}(H)$ in [4]:

$$
U_{\rho}(A) U_{\rho}(B) \geq \frac{1}{4}|\operatorname{Tr}[\rho[A, B]]|^{2} .
$$

(c) 2013 Yanagi et al.; licensee Springer. This is an Open Access article distributed under the terms of the Creative Commons

Attribution License (http://creativecommons.org/licenses/by/2.0), which permits unrestricted use, distribution, and reproduction in any medium, provided the original work is properly cited. 
As stated in [4], the physical meaning of the quantity $U_{\rho}(H)$ can be interpreted as follows. For a mixed state $\rho$, the variance $V_{\rho}(H)$ has both classical mixture and quantum uncertainty. Also, the Wigner-Yanase skew information $I_{\rho}(H)$ represents a kind of quantum uncertainty [6,7]. Thus, the difference $V_{\rho}(H)-I_{\rho}(H)$ has a classical mixture so that we can regard that the quantity $U_{\rho}(H)$ has a quantum uncertainty excluding a classical mixture. Therefore, it is meaningful and suitable to study an uncertainty relation for a mixed state by the use of the quantity $U_{\rho}(H)$.

Recently, a one-parameter extension of the inequality (1) was given in [8]:

$$
U_{\rho, \alpha}(A) U_{\rho, \alpha}(B) \geq \alpha(1-\alpha)|\operatorname{Tr}[\rho[A, B]]|^{2},
$$

where

$$
U_{\rho, \alpha}(H) \equiv \sqrt{V_{\rho}(H)^{2}-\left(V_{\rho}(H)-I_{\rho, \alpha}(H)\right)^{2}},
$$

with the Wigner-Yanase-Dyson skew information $I_{\rho, \alpha}(H)$ defined by

$$
I_{\rho, \alpha}(H) \equiv \frac{1}{2} \operatorname{Tr}\left[\left(i\left[\rho^{\alpha}, H_{0}\right]\right)\left(i\left[\rho^{1-\alpha}, H_{0}\right]\right)\right]=\operatorname{Tr}\left[\rho H_{0}^{2}\right]-\operatorname{Tr}\left[\rho^{\alpha} H_{0} \rho^{1-\alpha} H_{0}\right] .
$$

It is notable that the convexity of $I_{\rho, \alpha}(H)$ with respect to $\rho$ was successfully proven by Lieb in [9]. The further generalization of the Heisenberg-type uncertainty relation on $U_{\rho}(H)$ has been given in [10] using the generalized Wigner-Yanase-Dyson skew information introduced in [11]. Recently, it is shown that these skew informations are connected to special choices of quantum Fisher information in [12]. The family of all quantum Fisher informations is parametrized by a certain class of operator monotone functions $\mathcal{F}_{\text {op }}$ which were justified in [13]. The Wigner-Yanase skew information and Wigner-Yanase-Dyson skew information are given by the following operator monotone functions:

$$
\begin{aligned}
& f_{\mathrm{WY}}(x)=\left(\frac{\sqrt{x}+1}{2}\right)^{2}, \\
& f_{\mathrm{WYD}}(x)=\alpha(1-\alpha) \frac{(x-1)^{2}}{\left(x^{\alpha}-1\right)\left(x^{1-\alpha}-1\right)}, \alpha \in(0,1),
\end{aligned}
$$

respectively. In particular, the operator monotonicity of the function $f_{\mathrm{WYD}}$ was proved in [14] (see also [15]). On the other hand, the uncertainty relation related to the WignerYanase skew information was given by Luo [4], and the uncertainty relation related to the Wigner-Yanase-Dyson skew information was given by Yanagi [8]. In this paper, we generalize these uncertainty relations to the uncertainty relations related to quantum Fisher informations by using (generalized) metric adjusted skew information or correlation measure.

\section{Operator monotone functions}

Let $M_{n}(\mathbb{C})$ (respectively $M_{n, s a}(\mathbb{C})$ ) be the set of all $n \times n$ complex matrices (respectively all $n \times n$ self-adjoint matrices), endowed with the Hilbert-Schmidt scalar product $\langle A, B\rangle=$ $\operatorname{Tr}\left(A^{*} B\right)$. Let $M_{n,+}(\mathbb{C})$ be the set of strictly positive elements of $M_{n}(\mathbb{C})$ and $M_{n,+, 1}(\mathbb{C})$ be the set of stricly positive density matrices, that is $M_{n,+, 1}(\mathbb{C})=\left\{\rho \in M_{n}(\mathbb{C}) \mid \operatorname{Tr} \rho=1\right.$, $\rho>0\}$. If it is not otherwise specified, from now on, we shall treat the case of faithful states, that is $\rho>0$.

A function $f:(0,+\infty) \rightarrow \mathbb{R}$ is said to be operator monotone if, for any $n \in \mathbb{N}$ and $A, B \in M_{n,+}(\mathbb{C})$ such that $0 \leq A \leq B$, the inequalities $0 \leq f(A) \leq f(B)$ hold. An operator monotone function is said to be symmetric if $f(x)=x f\left(x^{-1}\right)$ and normalized if $f(1)=1$. 
Definition 1. $\mathcal{F}_{o p}$ is the class of functions $f:(0,+\infty) \rightarrow(0,+\infty)$ such that

1. $f(1)=1$,

2. $t f\left(t^{-1}\right)=f(t)$,

3. $f$ is operator monotone.

Example 1. Examples of elements of $\mathcal{F}_{o p}$ are given by the following list:

$$
\begin{aligned}
& f_{\mathrm{RLD}}(x)=\frac{2 x}{x+1}, f_{\mathrm{WY}}(x)=\left(\frac{\sqrt{x}+1}{2}\right)^{2}, \\
& f_{\mathrm{BKM}}(x)=\frac{x-1}{\log x}, f_{\mathrm{SLD}}(x)=\frac{x+1}{2}, \\
& f_{\mathrm{WYD}}(x)=\alpha(1-\alpha) \frac{(x-1)^{2}}{\left(x^{\alpha}-1\right)\left(x^{1-\alpha}-1\right)}, \alpha \in(0,1) .
\end{aligned}
$$

Remark 1. Any $f \in \mathcal{F}_{\text {op }}$ satisfies

$$
\frac{2 x}{x+1} \leq f(x) \leq \frac{x+1}{2}, x>0 .
$$

For $f \in \mathcal{F}_{o p}$, define $f(0)=\lim _{x \rightarrow 0} f(x)$. We introduce the sets of regular and nonregular functions

$$
\mathcal{F}_{o p}^{r}=\left\{f \in \mathcal{F}_{o p} \mid f(0) \neq 0\right\}, \quad \mathcal{F}_{o p}^{n}=\left\{f \in \mathcal{F}_{o p} \mid f(0)=0\right\}
$$

and notice that trivially $\mathcal{F}_{o p}=\mathcal{F}_{o p}^{r} \cup \mathcal{F}_{o p}^{n}$.

Definition 2. For $f \in \mathcal{F}_{o p}^{r}$, we set

$$
\tilde{f}(x)=\frac{1}{2}\left[(x+1)-(x-1)^{2} \frac{f(0)}{f(x)}\right], x>0 .
$$

Theorem 1. ([12,16,17]) The correspondence $f \rightarrow \tilde{f}$ is a bijection between $\mathcal{F}_{o p}^{r}$ and $\mathcal{F}_{o p}^{n}$.

\section{Metric adjusted skew information and correlation measure}

In the Kubo-Ando theory of matrix means, one associates a mean to each operator monotone function $f \in \mathcal{F}_{o p}$ by the formula

$$
m_{f}(A, B)=A^{1 / 2} f\left(A^{-1 / 2} B A^{-1 / 2}\right) A^{1 / 2},
$$

where $A, B \in M_{n,+}(\mathbb{C})$. Using the notion of matrix means, one may define the class of monotone metrics (also called quantum Fisher informations) by the following formula:

$$
\langle A, B\rangle_{\rho, f}=\operatorname{Tr}\left(A \cdot m_{f}\left(L_{\rho}, R_{\rho}\right)^{-1}(B)\right),
$$

where $L_{\rho}(A)=\rho A, R_{\rho}(A)=A \rho$. In this case, one has to think of $A, B$ as tangent vectors to the manifold $M_{n,+, 1}(\mathbb{C})$ at the point $\rho$ (see $\left.[12,13]\right)$.

Definition 3. For $A, B \in M_{n, s a}(\mathbb{C})$ and $\rho \in M_{n,+, 1}(\mathbb{C})$, we define the following quantities:

$$
\operatorname{Corr}{ }_{\rho}^{f}(A, B)=\operatorname{Tr}[\rho A B]-\operatorname{Tr}\left[A \cdot m_{\tilde{f}}\left(L_{\rho}, R_{\rho}\right) B\right],
$$




$$
\begin{aligned}
& \operatorname{Corr}_{\rho}^{s(f)}(A, B)=\frac{f(0)}{2}\langle i[\rho, A], i[\rho, B]\rangle_{\rho, f}, \\
& I_{\rho}^{f}(A)=\operatorname{Corr}_{\rho}^{f}(A, A), \\
& C_{\rho}^{f}(A, B)=\operatorname{Tr}\left[A \cdot m_{f}\left(L_{\rho}, R_{\rho}\right) B\right], \\
& C_{\rho}^{f}(A)=C_{\rho}^{f}(A, A), \\
& U_{\rho}^{f}(A)=\sqrt{V_{\rho}(A)^{2}-\left(V_{\rho}(A)-I_{\rho}^{f}(A)\right)^{2},}
\end{aligned}
$$

The quantity $I_{\rho}^{f}(A)$ is known as metric adjusted skew information [18], and the metric adjusted correlation measure $\operatorname{Corr}_{\rho}^{f}(A, B)$ was also previously defined in [18].

Then we have the following proposition.

Proposition 1. ([16,19]) For $A, B \in M_{n, s a}(\mathbb{C})$ and $\rho \in M_{n,+, 1}(\mathbb{C})$, we have the following relations, where we put $A_{0}=A-\operatorname{Tr}[\rho A] I$ and $B_{0}=B-\operatorname{Tr}[\rho B] I$ :

$$
\begin{gathered}
\text { 1. } I_{\rho}^{f}(A)=I_{\rho}^{f}\left(A_{0}\right)=\operatorname{Tr}\left[\rho A_{0}^{2}\right]-\operatorname{Tr}\left[A_{0} \cdot m_{\tilde{f}}\left(L_{\rho}, R_{\rho}\right) A_{]}=V_{\rho}(A)-C_{\rho}^{\tilde{f}}\left(A_{0}\right),\right. \\
\text { 2. } J_{\rho}^{f}(A)=\operatorname{Tr}\left[\rho A_{0}^{2}\right]+\operatorname{Tr}\left[A_{0} \cdot m_{\tilde{f}}\left(L_{\rho}, R_{\rho}\right) A_{0}\right]=V_{\rho}(A)+C_{\rho}^{\tilde{f}}\left(A_{0}\right), \\
\text { 3. } \quad 0 \leq I_{\rho}^{f}(A) \leq U_{\rho}^{f}(A) \leq V_{\rho}(A), \\
\text { 4. } U_{\rho}^{f}(A)=\sqrt{I_{\rho}^{f}(A) \cdot J_{\rho}^{f}(A),} \\
\text { 5. } \quad \operatorname{Corr}_{\rho}^{f}(A, B)=\operatorname{Corr}_{\rho}^{f}\left(A_{0}, B_{0}\right)=\operatorname{Tr}\left[\rho A_{0} B_{0}\right]-\operatorname{Tr}\left[A_{0} \cdot m_{\tilde{f}}\left(L_{\rho}, R_{\rho}\right) B_{0}\right], \\
\text { 6. } \quad \operatorname{Corr}_{\rho}^{s(f)}(A, B)=\operatorname{Corr}_{\rho}^{s(f)}\left(A_{0}, B_{0}\right) \\
=\frac{1}{2} \operatorname{Tr}\left[\rho A_{0} B_{0}\right]+\frac{1}{2} \operatorname{Tr}\left[\rho B_{0} A_{0}\right]-\operatorname{Tr}\left[A_{0} \cdot m_{\tilde{f}}\left(L_{\rho}, R_{\rho}\right) B_{0}\right] \\
=\frac{1}{2} \operatorname{Tr}\left[\rho A_{0} B_{0}\right]+\frac{1}{2} \operatorname{Tr}\left[\rho B_{0} A_{0}\right]-C_{\rho}^{\tilde{f}}\left(A_{0}, B_{0}\right) .
\end{gathered}
$$

Now we modify the uncertainty relation given by [20].

Theorem 2. For $f \in \mathcal{F}_{\text {op }}^{r}$, it holds

$$
I_{\rho}^{f}(A) \cdot I_{\rho}^{f}(B) \geq\left|\operatorname{Corr}_{\rho}^{s(f)}(A, B)\right|^{2},
$$

where $A, B \in M_{n, s a}(\mathbb{C})$ and $\rho \in M_{n,+, 1}(\mathbb{C})$.

Remark 2 . Since Theorem 2 is easily given by using the Schwarz inequality, we omit the proof. In [20] we gave the uncertainty relation

$$
U_{\rho}^{f}(A) \cdot U_{\rho}^{f}(B) \geq 4 f(0)\left|\operatorname{Corr}_{\rho}^{s(f)}(A, B)\right|^{2} .
$$

But since $4 f(0) \leq 1$ and $I_{\rho}^{f}(A) \leq U_{\rho}^{f}(A)$, it is easily given by Theorem 2 .

Theorem 3. ([20,21]) For $f \in \mathcal{F}_{o p}^{r}$, if

$$
\frac{x+1}{2}+\tilde{f}(x) \geq 2 f(x),
$$

then it holds

$$
\begin{aligned}
& U_{\rho}^{f}(A) \cdot U_{\rho}^{f}(B) \geq f(0)|\operatorname{Tr}(\rho[A, B])|^{2}, \\
& U_{\rho}^{f}(A) \cdot U_{\rho}^{f}(B) \geq 4 f(0)\left|\operatorname{Corr}_{\rho}^{f}(A, B)\right|^{2},
\end{aligned}
$$


where $A, B \in M_{n, s a}(\mathbb{C})$ and $\rho \in M_{n,+, 1}(\mathbb{C})$.

Remark 3. Though we cannot use the Schwarz inequality, we can get (4) in Theorem 3 by modifying the proof given by [20].

By putting

$$
f_{\mathrm{WYD}}(x)=\alpha(1-\alpha) \frac{(x-1)^{2}}{\left(x^{\alpha}-1\right)\left(x^{1-\alpha}-1\right)}, \quad \alpha \in(0,1),
$$

we obtain the following uncertainty relation.

Corollary 1. For $A, B \in M_{n, s a}(\mathbb{C})$ and $\rho \in M_{n,+, 1}(\mathbb{C})$,

$$
\begin{aligned}
& U_{\rho}^{f_{W Y D}}(A) \cdot U_{\rho}^{f_{W Y D}}(B) \geq \alpha(1-\alpha)|\operatorname{Tr}(\rho[A, B])|^{2}, \\
& U_{\rho}^{f_{W Y D}}(A) \cdot U_{\rho}^{f_{W Y D}}(B) \geq 4 \alpha(1-\alpha)\left|\operatorname{Corr}_{\rho}^{f_{W Y D}}(A, B)\right|^{2},
\end{aligned}
$$

where

$$
\operatorname{Corr}_{\rho}^{f_{W Y D}}(A, B)=\operatorname{Tr}\left[\rho A_{0} B_{0}\right]-\frac{1}{2} \operatorname{Tr}\left[\rho^{\alpha} A_{0} \rho^{1-\alpha} B_{0}\right]-\frac{1}{2} \operatorname{Tr}\left[\rho^{\alpha} B_{0} \rho^{1-\alpha} A_{0}\right] .
$$

Remark 4. Even if (2) does not necessarily hold, then

$$
\begin{aligned}
& U_{\rho}^{f}(A) \cdot U_{\rho}^{f}(B) \geq f(0)^{2} \mid \operatorname{Tr}\left[\left.(\rho[A, B])\right|^{2},\right. \\
& U_{\rho}^{f}(A) \cdot U_{\rho}^{f}(B) \geq 4 f(0)^{2}\left|\operatorname{Corr}_{\rho}^{f}(A, B)\right|^{2},
\end{aligned}
$$

where $A, B \in M_{n, s a}(\mathbb{C})$ and $\rho \in M_{n,+, 1}(\mathbb{C})$. Since $f(0)<1$, it is easy to show that (5) and (6) are weaker than (3) and (4), respectively.

\section{Generalized metric adjusted skew information and correlation measure}

We give some generalizations of Heisenberg or Schrd̈inger uncertainty relations which include Theorem 3 as corollary.

Definition 4. ([22]) Let $g, f \in \mathcal{F}_{o p}^{r}$ satisfy

$$
g(x) \geq k \frac{(x-1)^{2}}{f(x)}
$$

for some $k>0$. We define

$$
\Delta_{g}^{f}(x)=g(x)-k \frac{(x-1)^{2}}{f(x)} \in \mathcal{F}_{o p} .
$$

Definition 5. For $A, B \in M_{n, s a}(\mathbb{C})$ and $\rho \in M_{n,+, 1}(\mathbb{C})$, we define the following quantities:

$$
\begin{aligned}
& \operatorname{Corr}_{\rho}^{s(g, f)}(A, B)=k\langle i[\rho, A], i[\rho, B]\rangle_{\rho, f}, \\
& I_{\rho}^{(g, f)}(A)=\operatorname{Corr}_{\rho}^{s(g, f)}(A, A), \\
& C_{\rho}^{f}(A, B)=\operatorname{Tr}\left[A \cdot m_{f}\left(L_{\rho}, R_{\rho}\right) B\right], \\
& C_{\rho}^{f}(A)=C_{\rho}^{f}(A, A),
\end{aligned}
$$




$$
U_{\rho}^{(g, f)}(A)=\sqrt{\left(C_{\rho}^{g}(A)+C_{\rho}^{\Delta_{g}^{f}}(A)\right)\left(C_{\rho}^{g}(A)-C_{\rho}^{\Delta_{g}^{f}}(A)\right)} .
$$

The quantity $I_{\rho}^{(g, f)}(A)$ and $\operatorname{Corr}_{\rho}^{s(g, f)}(A, B)$ are said to be generalized metric adjusted skew information and generalized metric adjusted correlation measure, respectively.

Then we have the following proposition.

Proposition 2. For $A, B \in M_{n, s a}(\mathbb{C})$ and $\rho \in M_{n,+, 1}(\mathbb{C})$, we have the following relations, where we put $A_{0}=A-\operatorname{Tr}[\rho A] I$ and $B_{0}=B-\operatorname{Tr}[\rho B] I$ :

$$
\begin{array}{ll}
\text { 1. } & I_{\rho}^{(g, f)}(A)=I_{\rho}^{(g, f)}\left(A_{0}\right)=C_{\rho}^{g}\left(A_{0}\right)-C_{\rho}^{\Delta_{g}^{f}}\left(A_{0}\right), \\
\text { 2. } & J_{\rho}^{(g, f)}(A)=C_{\rho}^{g}\left(A_{0}\right)+C_{\rho}^{\Delta_{g}^{f}}\left(A_{0}\right), \\
\text { 3. } & U_{\rho}^{(g, f)}(A)=\sqrt{I_{\rho}^{(g, f)}(A) \cdot J_{\rho}^{(g, f)}(A)}, \\
\text { 4. } & \operatorname{Corr}_{\rho}^{s(g, f)}(A, B)=\operatorname{Corr}_{\rho}^{s(g, f)}\left(A_{0}, B_{0}\right) .
\end{array}
$$

Theorem 4. For $f \in \mathcal{F}_{o p}^{r}$, it holds

$$
I_{\rho}^{(g, f)}(A) \cdot I_{\rho}^{(g, f)}(B) \geq\left|\operatorname{Corr}_{\rho}^{s(g, f)}(A, B)\right|^{2},
$$

where $A, B \in M_{n, s a}(\mathcal{C})$ and $\rho \in M_{n,+, 1}(\mathbb{C})$.

Proof of Theorem 4. We define for $X, Y \in M_{n}(\mathbb{C})$

$$
\operatorname{Corr}_{\rho}^{s(g, f)}(X, Y)=k\langle i[\rho, X], i[\rho, Y]\rangle_{\rho, f} \text {. }
$$

Since

$$
\begin{aligned}
\operatorname{Corr}_{\rho}^{s(g, f)}(X, Y) & =k \operatorname{Tr}\left((i[\rho, X])^{*} m_{f}\left(L_{\rho}, R_{\rho}\right)^{-1} i[\rho, Y]\right) \\
& =k \operatorname{Tr}\left(\left(i\left(L_{\rho}-R_{\rho}\right) X\right)^{*} m_{f}\left(L_{\rho}, R_{\rho}\right)^{-1} i\left(L_{\rho}-R_{\rho}\right) Y\right) \\
& =\operatorname{Tr}\left(X^{*} m_{g}\left(L_{\rho}, R_{\rho}\right) Y\right)-\operatorname{Tr}\left(X^{*} m_{\Delta_{g}^{f}}\left(L_{\rho}, R_{\rho}\right) Y\right),
\end{aligned}
$$

it is easy to show that $\operatorname{Corr}_{\rho}^{s(g, f)}(X, Y)$ is an inner product in $M_{n}(\mathbb{C})$. Then we can get the result by using the Schwarz inequality.

Theorem 5. For $f \in \mathcal{F}_{o p}^{r}$, if

$$
g(x)+\Delta_{g}^{f}(x) \geq \ell f(x)
$$

for some $\ell>0$, then it holds

$$
U_{\rho}^{(g, f)}(A) \cdot U_{\rho}^{(g, f)}(B) \geq k \ell|\operatorname{Tr}(\rho[A, B])|^{2},
$$

where $A, B \in M_{n, s a}(\mathbb{C})$ and $\rho \in M_{n,+, 1}(\mathbb{C})$.

In order to prove Theorem 5 , we need the following lemmas.

Lemma 1. If (7) and (8) are satisfied, then we have the following inequality:

$$
m_{g}(x, y)^{2}-m_{\Delta_{g}^{f}}(x, y)^{2} \geq k \ell(x-y)^{2} .
$$


Proof of Lemma 1. By (7) and (8), we have

$$
\begin{gathered}
m_{\Delta_{g}^{f}}(x, y)=m_{g}(x, y)-k \frac{(x-y)^{2}}{m_{f}(x, y)}, \\
m_{g}(x, y)+m_{\Delta_{g}^{f}}(x, y) \geq \ell m_{f}(x, y) .
\end{gathered}
$$

Therefore, by (10) and (11),

$$
\begin{aligned}
& m_{g}(x, y)^{2}-m_{\Delta_{g}^{f}}(x, y)^{2} \\
= & \left\{m_{g}(x, y)-m_{\Delta_{g}^{f}}(x, y)\right\}\left\{m_{g}(x, y)+m_{\Delta_{g}^{f}}(x, y)\right\} \\
\geq & k \frac{(x-y)^{2}}{m_{f}(x, y)} \ell m_{f}(x, y) \\
= & k \ell(x-y)^{2} .
\end{aligned}
$$

We have the following expressions for the quantities $I_{\rho}^{(g, f)}(A), J_{\rho}^{(g, f)}(A), U_{\rho}^{(g, f)}(A)$, and $\operatorname{Corr}_{\rho}^{s(g, f)}(A, B)$ by using Proposition 2 and a mean $m_{\Delta_{g}^{f}}$.

Lemma 2. Let $\left\{\left|\phi_{1}\right\rangle,\left|\phi_{2}\right\rangle, \ldots,\left|\phi_{n}\right\rangle\right\}$ be a basis of eigenvectors of $\rho$, corresponding to the eigenvalues $\left\{\lambda_{1}, \lambda_{2}, \ldots, \lambda_{n}\right\}$. We put $a_{j k}=\left\langle\phi_{j}\left|A_{0}\right| \phi_{k}\right\rangle, b_{j k}=\left\langle\phi_{j}\left|B_{0}\right| \phi_{k}\right\rangle$, where $A_{0} \equiv A-$ $\operatorname{Tr}[\rho A] I$ and $B_{0} \equiv B-\operatorname{Tr}[\rho B] I$ for $A, B \in M_{n, s a}(\mathbb{C})$ and $\rho \in M_{n,+, 1}(\mathbb{C})$. Then we have

$$
\begin{aligned}
I_{\rho}^{(g, f)}(A) & =\sum_{j, k} m_{g}\left(\lambda_{j}, \lambda_{k}\right) a_{j k} a_{k j}-\sum_{j, k} m_{\Delta_{g}^{f}}\left(\lambda_{j}, \lambda_{k}\right) a_{j k} a_{k j} \\
& =2 \sum_{j<k}\left\{\left(m_{g}\left(\lambda_{j}, \lambda_{k}\right)-m_{\Delta_{g}^{f}}\left(\lambda_{j}, \lambda_{k}\right)\right\}\left|a_{j k}\right|^{2},\right. \\
J_{\rho}^{(g, f)}(A) & =\sum_{j, k} m_{g}\left(\lambda_{j}, \lambda_{k}\right) a_{j k} a_{k j}+\sum_{j, k} m_{\Delta_{g}^{f}}\left(\lambda_{j}, \lambda_{k}\right) a_{j k} a_{k j} \\
& \geq 2 \sum_{j<k}\left\{m_{g}\left(\lambda_{j}, \lambda_{k}\right)+m_{\Delta_{g}^{f}}\left(\lambda_{j}, \lambda_{k}\right)\right\}\left|a_{j k}\right|^{2}, \\
U_{\rho}^{(g, f)}(A)^{2}= & \left(\sum_{j, k} m_{g}\left(\lambda_{j}, \lambda_{k}\right)\left|a_{j k}\right|^{2}\right)^{2}-\left(\sum_{j, k} m_{\Delta_{g}^{f}}\left(\lambda_{j}, \lambda_{k}\right)\left|a_{j k}\right|^{2}\right)^{2},
\end{aligned}
$$

and

$$
\begin{aligned}
& \operatorname{Corr}_{\rho}^{s(g, f)}(A, B) \\
= & \sum_{j, k} m_{g}\left(\lambda_{j}, \lambda_{k}\right) a_{j k} b_{k j}-\sum_{j, k} m_{\Delta_{g}^{f}}\left(\lambda_{j}, \lambda_{k}\right) a_{j k} b_{k j} \\
= & \sum_{j<k}\left(m_{g}\left(\lambda_{j}, \lambda_{k}\right)-m_{\Delta_{g}^{f}}\left(\lambda_{j}, \lambda_{k}\right)\right) a_{j k} b_{k j}+\sum_{j<k}\left(m_{g}\left(\lambda_{k}, \lambda_{j}\right)-m_{\Delta_{g}^{f}}\left(\lambda_{k}, \lambda_{j}\right)\right) a_{k j} b_{j k} .
\end{aligned}
$$

We are now in a position to prove Theorem 5.

Proof of Theorem 5. At first we prove (9). Since

$$
\operatorname{Tr}(\rho[A, B])=\sum_{j, k}\left(\lambda_{j}-\lambda_{k}\right) a_{j k} b_{k j},
$$




$$
|\operatorname{Tr}(\rho[A, B])| \leq \sum_{j, k}\left|\lambda_{j}-\lambda_{k}\right|\left|a_{j k}\right|\left|b_{k j}\right| .
$$

Then by Lemma 1, we have

$$
\begin{aligned}
& k \ell|\operatorname{Tr}(\rho[A, B])|^{2} \\
\leq & \left\{\sum_{j, k} \sqrt{k \ell}\left|\lambda_{j}-\lambda_{k}\right|\left|a_{j k}\right|\left|b_{k j}\right|\right\}^{2} \\
\leq & \left\{\sum_{j, k}\left(m_{g}\left(\lambda_{j}, \lambda_{k}\right)^{2}-m_{\Delta_{g}^{f}}\left(\lambda_{j}, \lambda_{k}\right)^{2}\right)^{1 / 2}\left|a_{j k}\right|\left|b_{k j}\right|\right\}^{2} \\
\leq & \left\{\sum_{j, k}\left(m_{g}\left(\lambda_{j}, \lambda_{k}\right)-m_{\Delta_{g}^{f}}\left(\lambda_{j} u, \lambda_{k}\right)\right)\left|a_{j k}\right|^{2}\right\}\left\{\sum_{j, k}\left(m_{g}\left(\lambda_{j}, \lambda_{k}\right)+m_{\Delta_{g}^{f}}\left(\lambda_{j}, \lambda_{k}\right)\right)\left|b_{k j}\right|^{2}\right\} \\
= & I_{\rho}^{(g, f)}(A) J_{\rho}^{(g, f)}(B) .
\end{aligned}
$$

By a similar way, we also have

$$
I_{\rho}^{(g, f)}(B) J_{\rho}^{(g, f)}(A) \geq k \ell|\operatorname{Tr}(\rho[A, B])|^{2} .
$$

Hence, we have the desired inequality (9).

We give some examples satisfying the condition (8).

Example 2. Let

$$
\begin{aligned}
& g(x)=\frac{x+1}{2} \\
& f(x)=\alpha(1-\alpha) \frac{(x-1)^{2}}{\left(x^{\alpha}-1\right)\left(x^{1-\alpha}-1\right)}, \alpha \in(0,1), \\
& k=\frac{f(0)}{2}=\frac{\alpha(1-\alpha)}{2}, \ell=2 .
\end{aligned}
$$

Then

$$
g(x)+\Delta_{g}^{f}(x) \geq 2 f(x) .
$$

Proof of Example 2. In $[10,21]$ we give

$$
\left(x^{2 \alpha}-1\right)\left(x^{2(1-\alpha)}-1\right) \geq 4 \alpha(1-\alpha)(x-1)^{2}
$$

for $x>0$ and $0 \leq \alpha \leq 1$. Then we have

$$
g(x)+\Delta_{g}^{f}(x) \geq 2 f(x) .
$$

\section{Example 3. Let}

$$
\begin{aligned}
& g(x)=\left(\frac{\sqrt{x}+1}{2}\right)^{2}, \\
& f(x)=\alpha(1-\alpha) \frac{(x-1)^{2}}{\left(x^{\alpha}-1\right)\left(x^{1-\alpha}-1\right)}, \alpha \in(0,1),
\end{aligned}
$$




$$
k=\frac{f(0)}{8}=\frac{\alpha(1-\alpha)}{8}, \quad \ell=\frac{3}{2} .
$$

Then

$$
g(x)+\Delta_{g}^{f}(x) \geq \frac{3}{2} f(x)
$$

holds for $0<\alpha<1$.

Proof of Example 3. Since

$$
\begin{aligned}
& \frac{1}{2}\left(\frac{1+\sqrt{x}}{2}\right)^{2}-\frac{1}{8}\left(x^{\alpha}-1\right)\left(x^{1-\alpha}-1\right) \\
= & \frac{1}{8}\left(x+2 \sqrt{x}+1-x-1+x^{\alpha}+x^{1-\alpha}\right) \\
= & \frac{1}{8}\left(2 \sqrt{x}+x^{\alpha}+x^{1-\alpha}\right) \\
= & \frac{1}{8}\left(x^{\alpha / 2}+x^{(1-\alpha) / 2}\right)^{2} \geq 0,
\end{aligned}
$$

we have

$$
2\left(\frac{1+\sqrt{x}}{2}\right)^{2} \geq \frac{1}{8}\left(x^{\alpha}-1\right)\left(x^{1-\alpha}-1\right)+\frac{3}{2}\left(\frac{1+\sqrt{x}}{2}\right)^{2} .
$$

Since

$$
\alpha(1-\alpha) \frac{(x-1)^{2}}{\left(x^{\alpha}-1\right)\left(x^{1-\alpha}-1\right)} \leq\left(\frac{1+\sqrt{x}}{2}\right)^{2}
$$

we have

$$
2\left(\frac{1+\sqrt{x}}{2}\right)^{2} \geq \frac{1}{8}\left(x^{\alpha}-1\right)\left(x^{1-\alpha}-1\right)+\frac{3}{2} \alpha(1-\alpha) \frac{(x-1)^{2}}{\left(x^{\alpha}-1\right)\left(x^{1-\alpha}-1\right)} .
$$

Then we have

$$
g(x)+\Delta_{g}^{f}(x) \geq \frac{3}{2} f(x)
$$

Example 4. Let

$$
\begin{aligned}
& g(x)=\left(\frac{x^{\gamma}+1}{2}\right)^{1 / \gamma} \quad\left(\frac{3}{4} \leq \gamma \leq 1\right), \\
& f(x)=\left(\frac{\sqrt{x}+1}{2}\right)^{2}, \\
& k=\frac{f(0)}{4}=\frac{1}{16}, \quad \ell=2 .
\end{aligned}
$$

Then $g(x)+\Delta_{g}^{f}(x) \geq 2 f(x)$.

In order to prove Example 4, we need the following lemma.

Lemma 3. For $x>0$, we set the function of $y$ as

$$
F(y) \equiv\left(\frac{1+x^{y}}{2}\right)^{1 / y} \text {. }
$$

Then $F(y)$ has following properties:

1. $F(y)$ is monotone increasing for $y \in \mathbb{R}$. 
2. $F(y)$ is convex for $y<0$.

3. $F(y)$ is concave for $y \geq 1 / 2$.

We give the proof of Lemma 3 in the Appendix.

Proof of Example 4. By Lemma 3,

$$
2\left(\frac{1+x^{3 / 4}}{2}\right)^{4 / 3} \geq \frac{1+x}{2}+\left(\frac{1+\sqrt{x}}{2}\right)^{2} .
$$

It follows from the monotonicity that

$$
\left(\frac{1+x^{y}}{2}\right)^{1 / y} \geq\left(\frac{1+x^{3 / 4}}{2}\right)^{4 / 3}
$$

for $y \in[3 / 4,1]$. Then

$$
2\left(\frac{1+x^{y}}{2}\right)^{1 / y} \geq \frac{1+x}{2}+\left(\frac{1+\sqrt{x}}{2}\right)^{2}
$$

for $y \in[3 / 4,1]$. Therefore, we have

$$
2\left(\frac{1+x^{y}}{2}\right)^{1 / y}-\left(\frac{\sqrt{x}-1}{2}\right)^{2} \geq 2\left(\frac{\sqrt{x}+1}{2}\right)^{2} .
$$

Hence, we have

$$
g(x)+\Delta_{g}^{f}(x) \geq 2 f(x)
$$

\section{Appendix}

Proof of Lemma 3.

(i) Since $F(y)>0$ for $x>0$ and $t \in \mathbb{R}$, it is sufficient to prove $\frac{d}{d y} \log F(y)>0$ for the proof of $F^{\prime}(y)>0$. We have

$$
\frac{d}{d y} \log F(y)=\frac{1}{y^{2}}\left(\log 2+\frac{x^{y} \log x^{y}}{1+x^{y}}-\log \left(1+x^{y}\right)\right) .
$$

Then we put

$$
G(r) \equiv(r+1) \log 2+r \log r-(r+1) \log (r+1),(r>0),
$$

where we put $x^{y} \equiv r>0$. From elementary calculations, we have $G(r) \geq G(1)=0$ which implies $\frac{d}{d y} \log F(y)>0$.

(ii) We firstly set $f(y) \equiv \log F(y)$. Since $F(y)>0$, we have only to prove $f^{\prime \prime}(y)>0$ for the proof of $F^{\prime \prime}(y)>0$. We set again $g(y) \equiv \frac{1+x^{y}}{2},(x>0, y<0)$. Then we have $\frac{d^{2}}{d y^{2}} \log g(y) \equiv \frac{x^{y}(\log x)^{2}}{\left(1+x^{y}\right)^{2}}>0$. In addition, by $f(y)=\frac{1}{y} \log g(y)$, we have

$$
f^{\prime}(y)=\frac{1}{y} \frac{g^{\prime}(y)}{g(y)}-\frac{1}{y^{2}} \log g(y)>0 .
$$

By $\frac{d^{2}}{d y^{2}} \log g(y)=\frac{g(y) g^{\prime \prime}(y)-\left\{g^{\prime}(y)\right\}^{2}}{g(y)^{2}}$, we have

$$
f^{\prime \prime}(y)=\frac{1}{y} \frac{g(y) g^{\prime \prime}(y)-\left\{g^{\prime}(y)\right\}^{2}}{g(y)^{2}}-\frac{2}{y^{2}} \frac{g^{\prime}(y)}{g(y)}+\frac{2}{y^{3}} \log g(y)=\frac{1}{y} \frac{d^{2}}{d y^{2}} \log g(y)-\frac{2}{y} f^{\prime}(y) .
$$


We prove $f^{\prime \prime}(y)>0$ for $y<0$. We calculate

$$
\begin{aligned}
f^{\prime \prime}(y) & =\frac{1}{y} \frac{x^{y}(\log x)^{2}}{\left(1+x^{y}\right)^{2}}-\frac{2}{y} \frac{1}{y^{2}}\left(\log 2+\frac{x^{y} \log x^{y}}{1+x^{y}}-\log \left(1+x^{y}\right)\right) \\
& =\frac{1}{y^{3}\left(1+x^{y}\right)^{2}}\left\{-2 x^{y}\left(1+x^{y}\right) \log x^{y}+x^{y}\left(\log x^{y}\right)^{2}+2\left(1+x^{y}\right)^{2} \log \frac{1+x^{y}}{2}\right\} .
\end{aligned}
$$

Thus, if we put

$$
h(y) \equiv-2 x^{y}\left(1+x^{y}\right) \log x^{y}+x^{y}\left(\log x^{y}\right)^{2}+2\left(1+x^{y}\right)^{2} \log \frac{1+x^{y}}{2}
$$

then we have only to prove $h(y)<0$ for $y<0$. Since we have $h(0)=0$, we have only to prove $h^{\prime}(y)>0$ for $y<0$. Here we have

$$
h^{\prime}(y)=-x^{y} \log x\left\{4 x^{y} \log x^{y}-\left(\log x^{y}\right)^{2}-4\left(1+x^{y}\right) \log \frac{1+x^{y}}{2}\right\} .
$$

If we set again

$$
l(t) \equiv 4 t \log t-(\log t)^{2}-4(t+1) \log \frac{t+1}{2},
$$

where we put $x^{y} \equiv t>0$, then we prove the following cases:

(a) If $x<1$ (i.e., $t>1$ ), then $l(t)>0$.

(b) If $x>1$ (i.e., $0<t<1$ ), then $l(t)<0$.

For case (a), we calculate

$$
l^{\prime}(t)=\frac{1}{t}(4 t \log 2+(4 t-2) \log t-4 t \log (t+1))
$$

and

$$
l^{\prime \prime}(t)=\frac{2\{(t+1) \log t+t-1\}}{t^{2}(t+1)}>0,(t>1) .
$$

Thus, we have $l^{\prime}(t) \geq l^{\prime}(1)=0$, and then we have $l(t) \geq l(1)=0$. For case (b), we easily find that

$$
l^{\prime \prime}(t)=\frac{2\{(t+1) \log t+t-1\}}{t^{2}(t+1)}<0,(0<t<1) .
$$

Thus, we have $l^{\prime}(t) \geq l^{\prime}(1)=0$, and then we have $l(t) \leq l(1)=0$.

(iii) We calculate

$$
\frac{d^{2}}{d y^{2}} F(y)=\frac{1}{y^{4}}\left(\frac{1+x^{y}}{2}\right)^{1 / y} h(x, y)
$$

where

$$
\begin{aligned}
h(x, y)= & (\log 2-2 y) \log 2+\frac{2 \log 2}{1+x^{y}}\left\{x^{y} \log x^{y}-\left(1+x^{y}\right) \log \left(1+x^{y}\right)\right\} \\
& +\frac{1}{\left(1+x^{y}\right)^{2}}\left\{x^{y} y^{2}\left(x^{y}+y\right)(\log x)^{2}\right\} \\
& -\frac{1}{\left(1+x^{y}\right)^{2}}\left\{2 x^{y}\left(1+x^{y}\right)\left(y+\log \left(1+x^{y}\right)\right) \log x^{y}\right\} \\
& +\left\{2 y+\log \left(1+x^{y}\right)\right\} \log \left(1+x^{y}\right) .
\end{aligned}
$$

We prove $h(x, y) \leq 0$ for $x>0$ and $y \geq 1 / 2$. Then we have

$$
\frac{d h(x, y)}{d x}=-\frac{x^{-1+y} y^{2} \log x}{\left(1+x^{y}\right)^{3}}\left\{\left(x^{y}(y-2)-y\right) \log x^{y}+2\left(1+x^{y}\right) \log \left(\frac{1+x^{y}}{2}\right)\right\} \text {. }
$$


Here we note that $\frac{d h(1, y)}{d x}=0$. We also put

$$
g(x, y)=\left\{x^{y}(-2+y)-y\right\} \log x^{y}+2\left(1+x^{y}\right) \log \left(\frac{1+x^{y}}{2}\right) .
$$

If we have $g(x, y) \geq 0$ for $x>0$ and $y \geq 1 / 2$, then we have $\frac{d h(x, y)}{d x} \geq 0$ for $0<x \leq 1$ and $\frac{d h(x, y)}{d x} \leq 0$ for $x \geq 1$. Thus, we then obtain $h(x, y) \leq h(1, y)=0$ for $y \geq 1 / 2$, due to $\frac{d h(1, y)}{d x}=0$. Therefore, we have only to prove $g(x, y) \geq 0$ for $x>0$ and $y \geq 1 / 2$.

(a) For the case $0<x \leq 1$, we have

$$
\frac{d g(x, y)}{d x}=\frac{y}{x}\left\{y\left(x^{y}-1\right)+(y-2) x^{y} \log x^{y}+2 x^{y} \log \left(\frac{x^{y}+1}{2}\right)\right\} .
$$

Since $g(1, y)=0$, if we prove $\frac{d g(x, y)}{d x} \leq 0$, then we can prove $g(x, y) \geq$ $g(1, y)=0$ for $y \geq 1 / 2$ and $0<x \leq 1$. Since we have the relations

$$
\frac{x-1}{\sqrt{x}} \leq \log x \leq \frac{2(x-1)}{x+1} \leq 0
$$

for $0<x \leq 1$, we calculate

$$
\begin{aligned}
& y\left(x^{y}-1\right)+(y-2) x^{y} \log x^{y}+2 x^{y} \log \left(\frac{x^{y}+1}{2}\right) \\
& \leq y\left(x^{y}-1\right)+(y-2) x^{y} \frac{\left(x^{y}-1\right)}{x^{y / 2}}+2 x^{y} \frac{2\left(\frac{x^{y}+1}{2}-1\right)}{\frac{x^{y}+1}{2}+1} \\
& =\frac{x^{y}-1}{x^{y}+3}\left\{3(y-2) x^{y / 2}+(y-2) x^{3 y / 2}+3 y+(y+4) x^{y}\right\} .
\end{aligned}
$$

Thus, we have only to prove

$$
k(y) \equiv 3(y-2) x^{y / 2}+(y-2) x^{3 y / 2}+3 y+(y+4) x^{y} \geq 0
$$

for $0<x \leq 1$ and $y \geq 1 / 2$. Since it is trivial $k(y) \geq 0$ for $y \geq 2$, we assume $1 / 2 \leq y<2$ from here. To this end, we prove that $k_{1}(y) \equiv 3(y-2) x^{y / 2}+$ $(y-2) x^{3 y / 2}$ is monotone increasing for $1 / 2 \leq y<2$ and $k_{2}(y) \equiv 3 y+$ $(y+4) x^{y}$ is also monotone increasing for $1 / 2 \leq y<2$. We easily find that

$$
\frac{d k_{1}(y)}{d y}=\frac{1}{2} x^{y / 2}\left\{2\left(x^{y}+3\right)+3\left(x^{y}+1\right)(y-2) \log x\right\}>0,
$$

for $0<x \leq 1$ and $1 / 2 \leq y<2$.

We also have

$$
\frac{d k_{2}(y)}{d y}=x^{y}+3+(y+4) x^{y} \log x
$$

Here we prove $\frac{d k_{2}(y)}{d y} \geq 0$ for $0<x \leq 1$ and $1 / 2 \leq y<2$. We put again

$$
k_{3}(x) \equiv x^{y}+3+(y+4) x^{y} \log x,
$$

then we have

$$
\frac{d k_{3}(x)}{d x}=x^{-1+y}\{2(y+2)+y(y+4) \log x\} .
$$

Thus, we have

$$
\frac{d k_{3}(x)}{d x}=0 \Leftrightarrow x=\mathrm{e}^{-\frac{2(y+2)}{y(y+4)}} \equiv \alpha_{y} .
$$


Since $\frac{d k_{3}(x)}{d x}<0$ for $0<x<\alpha_{y}$ and $\frac{d k_{3}(x)}{d x}>0$ for $\alpha_{y}<x \leq 1$, we have

$$
k_{3}(x) \geq k_{3}\left(\alpha_{y}\right)=3-\frac{(y+4) \mathrm{e}^{-\frac{2(y+2)}{y+4}}}{y} \equiv k_{4}(y) .
$$

Since we have $\frac{d k_{4}(y)}{d y}=\frac{8(y+2) \mathrm{e}^{-\frac{2(y+2)}{y+4}}}{y^{2}(y+4)}>0$, the function $k_{4}(y)$ is monotone increasing for $y$. Thus, we have

$$
k_{3}(x) \geq k_{3}\left(\alpha_{y}\right)=3-\frac{(y+4) \mathrm{e}^{-\frac{2(y+2)}{y+4}}}{y} \equiv k_{4}(y) \geq k_{4}(1 / 2)=3-\frac{9}{\mathrm{e}^{10 / 9}}>0
$$

since $e^{10 / 9} \simeq 3.03773$. Therefore, $k_{2}(y)$ is also a monotone increasing function of $y$ for $0<x \leq 1$ and $1 / 2 \leq y<2$. Thus, $k(y)$ is monotone increasing for $y \geq 1 / 2$, and then we have

$$
k(y) \geq k(1 / 2)=-\frac{3}{2}\left(x^{1 / 4}-1\right)^{3} \geq 0 .
$$

(b) For the case $x \geq 1$, we firstly calculate

$$
\begin{aligned}
& \frac{d g(x, y)}{d y}=\left(x^{y}-1\right) \log x^{y} \\
& +\left\{y\left(x^{y}-1\right)+(y-2) x^{y} \log x^{y}+2 x^{y} \log \left(\frac{1+x^{y}}{2}\right)\right\} \log x .
\end{aligned}
$$

We put

$$
p(x, y) \equiv\left(x^{y}-1\right) y+x^{y}(y-2) \log x^{y}+2 x^{y} \log \left(\frac{1+x^{y}}{2}\right) .
$$

Then we calculate

$$
\begin{aligned}
& \frac{d p(x, y)}{d x}=\frac{y}{x+x^{1-y}}\left\{\left(1+x^{y}\right)(y-2) \log x^{y}\right. \\
& \left.+2\left(y\left(1+x^{y}\right)-1+\left(1+x^{y}\right) \log \left(\frac{1+x^{y}}{2}\right)\right)\right\} .
\end{aligned}
$$

Then we put

$$
q(x, y)=(y-2) \log x^{y}+2 \log \left(\frac{1+x^{y}}{2}\right)+2 y-\frac{2}{1+x^{y}} .
$$

We have

$$
\frac{d q(x, y)}{d y}=\frac{\left(\left(1+x^{y}\right)^{2} y-2\right) \log x+\left(1+x^{y}\right)^{2}\left(\log x^{y}+2\right)}{\left(1+x^{y}\right)^{2}}>0
$$

and then

$$
q(x, y) \geq q(x, 1 / 2)=1-\frac{2}{\sqrt{x}+1}+2 \log \left(\frac{1+\sqrt{x}}{2}\right)-\frac{3}{4} \log x .
$$

Since we find

$$
\frac{d q(x, 1 / 2)}{d x}=\frac{(\sqrt{x}+3)(\sqrt{x}-1)}{4 x(\sqrt{x}+1)^{2}} \geq 0
$$

for $x \geq 1$, we have $q(x, y) \geq q(x, 1 / 2) \geq q(1,1 / 2)=0$. Therefore, we have $\frac{d p(x, y)}{d x} \geq 0$, which implies $p(x, y) \geq p(1, y)=0$. Thus, we have $\frac{d g(x, y)}{d y} \geq 0$, and then we have $g(x, y) \geq g(x, 1 / 2)$, where

$$
g(x, 1 / 2)=-\frac{1}{2}\left(3 x^{1 / 2}+1\right) \log x^{1 / 2}+2\left(x^{1 / 2}+1\right) \log \left(\frac{x^{1 / 2}+1}{2}\right) .
$$


To prove $g(x, 1 / 2) \geq 0$ for $x \geq 1$ and $y \geq 1 / 2$, we put $x^{1 / 2} \equiv z \geq 1$ and

$$
r(z) \equiv-\frac{1}{2}(3 z+1) \log z+2(z+1) \log \left(\frac{z+1}{2}\right) .
$$

Since we have $r^{\prime \prime}(z)=\frac{(z-1)^{2}}{2 z^{2}(z+1)} \geq 0$ and

$$
r^{\prime}(z)=\frac{1}{2 z}\left\{z-1-3 z \log z+4 z \log \left(\frac{z+1}{2}\right)\right\},
$$

we have $r^{\prime}(1)=0$ and then we have $r^{\prime}(z) \geq 0$ for $z \geq 1$. Thus, we have $r(z) \geq 0$ for $z \geq 1$ by $r(1)=0$. Finally, we have $g(x, y) \geq g(x, 1 / 2) \geq 0$, for $x \geq 1$ and $y \geq 1 / 2$.

\section{Acknowledgements}

The first author (KY) was partially supported by JSPS KAKENHI Grant Number 23540208. The second author (SF) was partially supported by JSPS KAKENHI Grant Number 24540146.

\section{Author details \\ ${ }^{1}$ Graduate School of Science and Engineering, Yamaguchi University, Une, Yamaguchi 755-8611, Japan. ${ }^{2}$ College of Humanities and Science, Nihon University, Tokyo 156-8550, Japan. ${ }^{3}$ Faculty of Education, Bukkyo University, Kyoto} 603-8301, Japan.

\section{Received: 10 September 2013 Accepted: 24 October 2013}

Published: 11 November 2013

\section{References}

1. Heisenberg, W: Uber den anschaulichen Inhalt der quantummechanischen Kinematik und Mechanik. Zeitschrift für Physik. 43, 172-198 (1927)

2. Robertson, HP: The uncertainty principle. Phys. Rev. 34, 163-164 (1929)

3. Schrödinger, E: About Heisenberg uncertainty relation. Proc. Prussian Acad. Sci. Phys. Math. XIX, 293 (1930)

4. Luo, S: Heisenberg uncertainty relation for mixed states. Phys. Rev. A. 72, 042110 (2005)

5. Wigner, EP, Yanase, MM: Information content of distribution. Proc. Nat. Acad. Sci. 49, 910-918 (1963)

6. Luo, S, Zhang, Q: Informational distance on quantum-state space. Phys. Rev. A. 69, 032106 (2004)

7. Luo, S: Quantum versus classical uncertainty. Theor. Math. Phys. 143, 681-688 (2005)

8. Yanagi, K: Uncertainty relation on Wigner-Yanase-Dyson skew information. J. Math. Anal. Appl. 365, 12-18 (2010)

9. Lieb, EH: Convex trace functions and the Wigner-Yanase-Dyson conjecture. Adv. Math. 11, 267-288 (1973)

10. Yanagi, K: Uncertainty relation on generalized Wigner-Yanase-Dyson skew information. Linear Algebra Appl. 433, 1524-1532 (2010)

11. Cai, L, Luo, S: On convexity of generalized Wigner-Yanase-Dyson information. Lett. Math. Phys. 83, 253-264 (2008)

12. Gibilisco, P, Hansen, F, Isola, T: On a correspondence between regular and non-regular operator monotone functions. Linear Algebra Appl. 430, 2225-2232 (2009)

13. Petz, D: Monotone metrics on matrix spaces. Linear Algebra Appl. 244, 81-96 (1996)

14. Petz, D, Hasegawa, H: On the Riemannian metric of $\alpha$-entropies of density matrices. Lett. Math. Phys. 38, 221-225 (1996)

15. Furuta, T: Elementary proof of Petz-Hasegawa theorem. Lett. Math. Phys. 101, 355-359 (2012)

16. Gibilisco, P, Imparato, D, Isola, T: Uncertainty principle and quantum Fisher information, II. J. Math. Phys. 48, 072109 (2007)

17. Kubo, F, Ando, T: Means of positive linear operators. Math. Ann. 246, 205-224 (1980)

18. Hansen, F: Metric adjusted skew information. Proc. Nat. Acad. Sci. 105, 9909-9916 (2008)

19. Gibilisco, P, Isola, T: On a refinement of Heisenberg uncertainty relation by means of quantum Fisher information. J. Math. Anal. Appl. 375, 270-275 (2011)

20. Furuichi, S, Yanagi, K: Schrödinger uncertainty relation, Wigner-Yanase-Dyson skew information and metric adjusted correlation measure. J. Math. Anal. Appl. 388, 1147-1156 (2012)

21. Yanagi, K: Metric adjusted skew information and uncertainty relation. J. Math. Anal. Appl. 380, 888-892 (2011)

22. Gibilisco, P, Hiai, F, Petz, D: Quantum covariance, quantum Fisher information, and the uncertainty relations. IEEE Trans. Inf. Theory. 55, 439-443 (2009) 